\title{
AN AGENT-BASED SIMULATION OF A TUBERCULOSIS EPIDEMIC: UNDERSTANDING THE TIMING OF TRANSMISSION
}

\author{
Parastu Kasaie \\ Department of Operations, Business Analytics, and \\ Information Systems \\ University of Cincinnati, PO Box 210130 \\ Cincinnati, $\mathrm{OH} 45221-0130$, USA
}

David W. Dowdy

\author{
Bloomberg School of Public Health \\ The Johns Hopkins University \\ Baltimore, MD 21205, USA
}

\author{
W. David Kelton \\ Department of Operations, Business Analytics, and \\ Information Systems \\ University of Cincinnati, PO Box 210130 \\ Cincinnati, OH 45221-0130, USA
}

\begin{abstract}
Tuberculosis (TB) transmission is a key factor for disease-control policy, but the timing and distribution of transmission and the role of social contacts remain obscure. We develop an agent-based simulation of a TB epidemic in a single population, and consider a hierarchically structured contact network in three levels, typical of airborne diseases. The parameters are adopted from the literature, and the model is calibrated to a setting of high TB incidence. We model the dynamics of transmission at the individual level, and study the timing of secondary infections from a single source throughout the duration of the disease. We compare the patterns of transmission among different networks and discuss implications. Sensitivity analysis of outputs indicates the robustness of the results to variations in the parameter values.
\end{abstract}

\section{INTRODUCTION}

New and re-emerging infectious diseases pose an enormous burden on global health, and massive effort has been put into combating them. As an example, TB is a contagious bacterial infection responsible for 1.4 million deaths each year. About one third of the world's population is infected with TB, and close to 9 million new TB cases occur each year (CORE Group 2006). Despite the vast literature and ongoing research on disease evolution, however, our understanding of the transmission dynamics is incomplete, and many questions remain unanswered (Dowdy, Dye, and Cohen 2012).

TB is an airborne disease transmitted through infectious contact with an active case. TB transmission is one of the key determinants of epidemic severity, and has important implications for design, implementation, and scaling-up of control interventions (e.g., improved diagnosis, active case finding) that aim to reduce the rate of transmission. Unlike other airborne diseases such as influenza, however, TB transmission is not directly measurable, i.e., available diagnostic techniques cannot estimate the original timing of infection in diagnosed cases. TB also has a predilection for establishment of a latent state that is noninfectious and asymptomatic, but may progress to active, infectious disease at any time. As a result, one cannot reliably differentiate between primary infection with rapid progression to active disease, reinfection following a remote initial infection, or reactivation of a previous latent infection. Such limitations pose several challenges to the study of transmission dynamics across populations, including 
the lack of informative data to trace the chain of transmission across various contact networks in retrospective studies. The prospective following a cohort population, on the other hand, are prohibitively expensive and restricted by the time and budget constraints. In such settings, the relationships between the duration of disease, symptom burden, contact networks, diagnosis/treatment, and patients' infectiousness remain obscure (Dowdy, Dye, and Cohen 2012).

We propose an agent-based simulation (ABS) to study TB transmission dynamics and the role of various contact networks. Our model simulates the course of a TB epidemic across a single population and uses a hierarchical network of contacts in three levels, typical to the transmission of airborne diseases (Mossong et al. 2005). Parameters are chosen from the literature, and the model is calibrated to a setting of high TB incidence. We use our model to study the transmission dynamics at an individual level with regard to the timing and distribution of secondary infections from a single source. The average time for disease diffusion to reach $50 \%$ of infections at an individual level is estimated, and the timing patterns are compared among different networks. We perform sensitivity analysis of results with regard to multiple parameter values, and discuss the implications for TB control policy.

\section{BACKGROUND}

Modeling TB dynamics has a long history, including mathematical models and analytical techniques to describe and predict disease prevalence at the population level (Waaler, Gese, and Anderson 1962; Porco and Blower 1998; Castillo-Chavez and Song 2004). Analytical studies, however, are usually restricted by their simplifying assumptions regarding the population heterogeneity, network structure, and parameters uncertainty, and do not provide a realistic representation of transmission dynamics.

Simulation modeling of TB epidemics in human populations, on the other hand, has a shorter history. One group of studies uses system dynamics to model disease prevalence at the population level (Brewer et al. 1996; Atun et al. 2007). Following a top-down approach, these studies divide the population into different health states, and use transition rates to describe the disease's natural history. A system of differential equations is used to model disease prevalence through time. In comparison to the analytical approach, such studies apply a semi-Markov system in which transition rates can change with time, and are able to capture output uncertainty.

Other researchers have developed discrete-event simulation (DES) models of TB to evaluate the impact of new diagnostic tools (Langley et al. 2012), or to study more complex structure as in the coinfection of HIV/TB (Hughes, Currie, and Corbett 2006; Mellor, Currie, and Corbett 2011). The DES studies use a schedule of events that are executed in chronological order, and model disease transmission using random generation of Poisson distributions in each mixing group. The aggregate (top-bottom) modeling nature of DES, however, offers low flexibility for direct modeling of contacts (and transmission events) at the individual level, and restricts application of such models to complex social networks.

ABS is an alternative approach that models the system at the individual level and offers high flexibility to incorporate various modeling assumptions. ABS models have wide application in the social sciences (Epstein 1999, Macal and North 2010), and the models have been applied to study various infectious diseases such as influenza (Chao et al. 2010), smallpox (Longini et al. 2006), and HIV/AIDS (Alam, Meyer, and Norling 2008). The literature on application of ABS models in the study of TB, however, is quite limited. Espindola et al. (2011) use an ABS approach to study the emergence of drug-resistant TB due to treatment with antibiotics. They assume a lattice structure for spatial presentation of their population and model TB transmission through local and global interactions across the lattice. The lattice structure, however, does not provide a realistic representation of social settings and cannot be used to study transmission dynamics at the individual level.

We consider a model of transmission involving three contact networks that represent the main social relationships in transmission of an airborne disease. Our model simulates the stochastic contact events at an individual level, and enables us to study patterns of transmission across different networks. 


\section{METHOD}

Agents represent people in the population whose personal characteristics, social relationships, and health states are programmed at the individual level (Figure 1). The model initiates from an existing epidemic and is calibrated to a setting of high TB incidence. The model is developed using Anylogic (XJ Technologies 2012) and the extensions are coded in Java. A time step of one month is chosen to update the contact networks and model the transmission events, while the internal simulation time-step is very small (0.001 month) and the population dynamics are simulated on a closely continuous time-scale. Outputs are gathered at the end of each year, and the time horizon is 50 years. In this section, we briefly describe the model's population structure, contact network, and natural history of TB, and discuss our calibration procedure.

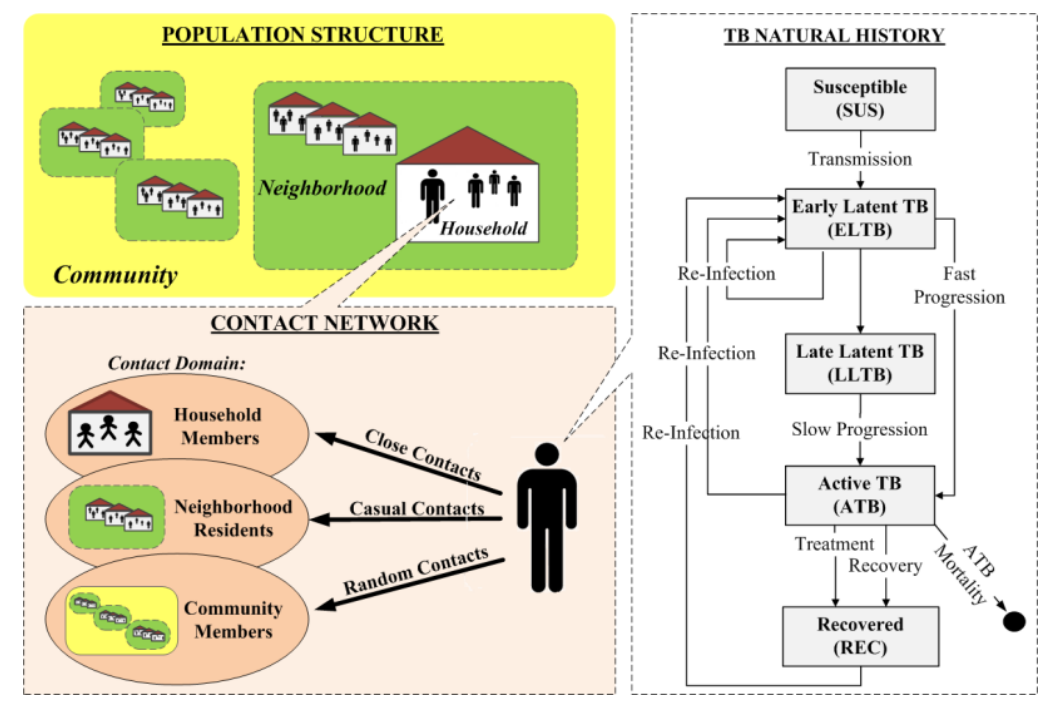

Figure 1: ABS model outline.

\subsection{Population Structure}

Following the literature on modeling of airborne infectious diseases (Raffalli, Sepkowitz, and Armstrong 1996) and using a similar approach to Chao et al. (2010), we consider a hierarchically structured population including households, neighborhoods (clusters of households) and communities (sets of neighborhoods). While the exact definition and size of these mixing groups are chosen arbitrarily, their inclusion in the model provides a realistic representation of the population structure, and enables us to model different transmission routes associated with each mixing group.

A household is the smallest and most intimate mixing group for transmission of airborne diseases (Verver et al. 2004; Chao et al. 2010). We assume that household size follows a triangular distribution, ranging from 1 to 10 with a mode of 5 people per family, which corresponds to low socioeconomic status typical of the high-incidence TB settings in India and Brazil. The natural mortality rate is defined at the individual level, and corresponds to a life expectancy of 73 years (Google 2010). We define the probability of child bearing for each family as a decreasing function of the family size such that the average population size remains constant, and the initial household-size distribution keeps its shape through the simulation (see Appendix A).

\subsection{TB Natural History}

We model TB natural history at the individual level using the five main TB health states (Figure 1). A person is assumed born in full health and susceptible to TB. Upon a successful transmission of the dis- 
ease, the person enters the early latent TB (ELTB) state for a period of five years, during which he endures a high chance of active TB development (fast progression rate). At the end of this period, the individual enters the late latent TB (LLTB) state, which can last for many years, and is associated with a much lower chance of symptom development (slow progression rate). Symptomatic patients with active $T B$ (ATB) are infectious and subject to an increased mortality rate. The ATB patients' infectiousness is modeled as a linear function of time, increasing during the first nine months of the disease from zero to a maximum infectiousness of $I_{\max }$, and staying unchanged for the rest of the disease. The $I_{\max }$ value is subsequently calibrated to the overall incidence rate (Section 3.4).

Active cases become recovered through spontaneous recovery or upon diagnosis and treatment. Recovered and latently infected individuals are subject to multiple re-infections, upon which they return to the ELTB state. Table 1 shows the disease parameters and their values.

Table 1: Parameters and Values

\begin{tabular}{|c|c|c|}
\hline Parameter & Value & Source/ Justification \\
\hline Population structure: & \multirow[b]{2}{*}{$50(40)$} & \multirow[b]{2}{*}{$\begin{array}{l}\text { Calibrated to provide the mean number } \\
\text { of contacts in each group (Appendix B) }\end{array}$} \\
\hline $\begin{array}{l}\text { Number of neighborhoods (Number } \\
\text { of families per neighborhood) }\end{array}$ & & \\
\hline Initial household-size distribution & Triangular $(1,5,10)$ & (United Nations Data System 2013) \\
\hline Life expectancy & 73 years & (Google 2010) \\
\hline \multicolumn{3}{|l|}{ Disease Natural History: } \\
\hline ATB mortality rate & 0.12 per year & (Tiemersma et al. 2011) \\
\hline Early latency duration & 5 years & (Dowdy et al. 2012) \\
\hline Fast progression rate & 0.03 per year & (Vynnycky and Fine 1997) \\
\hline Slow progression rate & 0.005 per year & (Horsburgh 2004) \\
\hline Recovery rate & 0.12 per year & (Tiemersma et al. 2011) \\
\hline Diagnosis \& treatment rate & 0.74 per year & $\begin{array}{l}\text { Calibrated to provide disease duration } \\
\text { of } 11 \text { months (WHO 2012) }\end{array}$ \\
\hline Latent immunity toward re-infection & 0.8 & (Andrews et al. 2012) \\
\hline
\end{tabular}

\subsection{Contact Network}

We define a three-layer contact network in association with our hierarchical population structure, referred to as close, casual, and random contacts. This is only an abstract representation of real social settings, but the lack of data on TB transmission restricts our ability to use a more sophisticated network. Nevertheless, the three-layer mixing structure enables us to represent the important routes of transmission detected in past TB breakouts (Raffalli, Sepkowitz, and Armstrong 1996; Classen et al. 1999), and contrast the dynamics of transmission over each network.

The three contact types represent the social relationships of any individual with the rest of the population (Figure 1). Each type is defined over a specific domain and among a sub-group of the population (e.g. household members). The individual's probability of engaging in a contact and the contact domain's size are calibrated to the average number of contacts in each network (Mossong et al. 2005). The contact duration (time delay between network updates representing the persistency of the social bounds) is chosen with regard to the definition of each category (e.g. close contacts are the most persistent type). Contact types are further differentiated by the effectiveness of contacts in transmission of the TB pathogen. The effectiveness parameters are calibrated to provide the given ratios of infections through each network (see Section 3.4). The infectious contacts are modeled at each time step, and the probability of disease transmission is computed with regard to the individual's infectiousness, immunity, and effectiveness of contact type. 
Close contacts, at the first level, represent the most frequent contact type among household members. On the second level, casual contacts model social relationships such as those among friends at a bar, neighbors at a store, or children at school. These contacts are less frequent and intimate than close contacts, and restricted to a specific network of related individuals including friends, coworkers, etc. (Raffalli, Sepkowitz, and Armstrong 1996; Classen et al. 1999). We restrict the domain of casual contacts to each neighborhood's residents, and assume a limited period of one year for the duration of contacts (Appendix B). Finally, random contacts are used to model encounters of people at places such as bus stops, museums, etc. Such contacts have the shortest duration (one month), and account for potential risk of transmission among non-related people in the whole community.

The suggested network structure can be further extended to a more detailed network (involving additional mixing groups such as schools, work places, etc.) so that it provides a more realistic view of social settings. The trade-off for developing such models, however, is lack of suitable data for calibration, as well as the computational expense of more extensive simulation runs.

\subsection{Calibration}

We model a community of 2000 households in 50 neighborhoods. The community size and its composition is tuned to provide the average number of contacts in each mixing group as described in Appendix B. Individuals are subject to a mortality rate so that some families may eventually die out from the model. We tune the probability of child bearing for each family such that the average population size remains constant (10200 people), and the initial family-size distribution keeps a stable shape through time (see Appendix A). The model is initiated from an existing epidemic, and is calibrated to provide an incidence rate of 120 per 100,000 people/year, representative of the current global TB incidence rate (WHO 2012).

In order to ensure the fidelity of the ABS model, we use a simplified instance of the simulation model with homogenous mixing structure as a benchmark, and align this model against a corresponding deterministic model of TB (Appendix C). Except for the subtle variation of simulation outputs due to stochasticity, the timing pattern and estimated values of compartment sizes at steady state are similar. Consequently, we extend the verified homogenous model to incorporate the heterogeneous network structure, and continue our analysis with this model.

Figure 2 shows the main ABS outputs over 150 years. The external infection ratio represents the proportion of disease incidence due to reactivation or progression among individuals who were latently infected at time zero (as a part of the existing epidemic). Since such infections did not originate within the simulation period, they cannot be used for the analysis of transmission. Moreover, due to the stochastic nature of the simulation model and disease dynamics, the annual incidence rate shows a non-stationary pattern during the first decades. We choose the simulation transient period such that the external infection ratio is less than $5 \%$, and the incidence rate has a stationary mean. We test the external infection ratio mean using a one-tailed t-test, and test the stationarity using multiple comparison tests of the incidence mean in consecutive 5-year periods after the transient time (using Bonferroni-adjusted paired t-tests). Consequently, we choose a transient period of 100 years for the simulation model, and start our analysis of the output in year 101 .
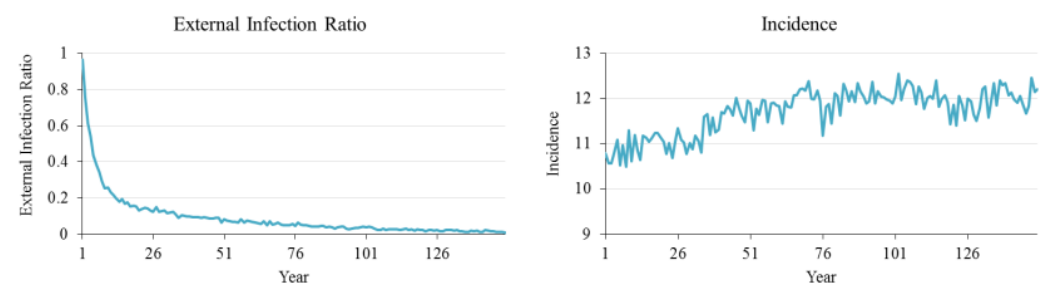

Figure 2: ABS output patterns. 
Furthermore, we follow the literature on the proportion of TB transmissions in different social settings, and let close contacts account for $20 \%$ of transmissions (Verver et al. 2004) while casual contacts account for $60 \%$ of transmission corresponding to $75 \%$ of non-household infections (Classen et al. 1999). The effectiveness parameters for each contact type are subsequently calibrated to provide the specified transmission ratios (Table 1). The main outputs of the simulation model including the disease progression measures are provided in Appendix D.

\section{RESULTS}

We use the calibrated ABS model to study the dynamics of transmission at the individual level and estimate the timing of secondary infections from a single source during the course of the disease. Figure 3a shows the average frequency of infections from a single individual with active TB throughout the course of infectiousness (i.e., from onset of infectiousness, defined as time zero, to treatment or death, the timing of which varies across individuals). The three curves are stratified by the type of contact, and show a similar general pattern: increasing during the initial months as the infectiousness increases, and then decreasing as the infectiousness period ends (e.g., due to recovery). In these figures, the maximum frequency of infections reflects the proportion of transmission due to each type of contact (e.g., higher peak for casual contacts reflects our model assumption that these contacts are responsible for more transmission events than close or random contacts). The different shapes of these curves, however, demonstrate that transmission due to close contact occurs earlier in the course of infection and declines more rapidly over time such that, among individuals with very long periods of infectiousness, random contacts actually become more important sources of transmission than close (household) contacts.

Figure $3 \mathrm{~b}$ shows the cumulative ratio of infections in each network: half of all transmission events due to close contacts occur within the first 9 months of infectiousness, whereas only about $35 \%$ of transmission to non-household contacts has occurred by that time. Such differences can be partially explained with regard to the relative size of each network and development of immunological protection from repeat infections after initial infection, which limits the number of potential infections. The timing of transmission to casual versus random contacts is remarkably similar, suggesting that, once contact networks reach a certain size (i.e., low probability of reinfection), the timing of infection approaches a limiting shape regardless of the assumed duration and intensity of the contact network.
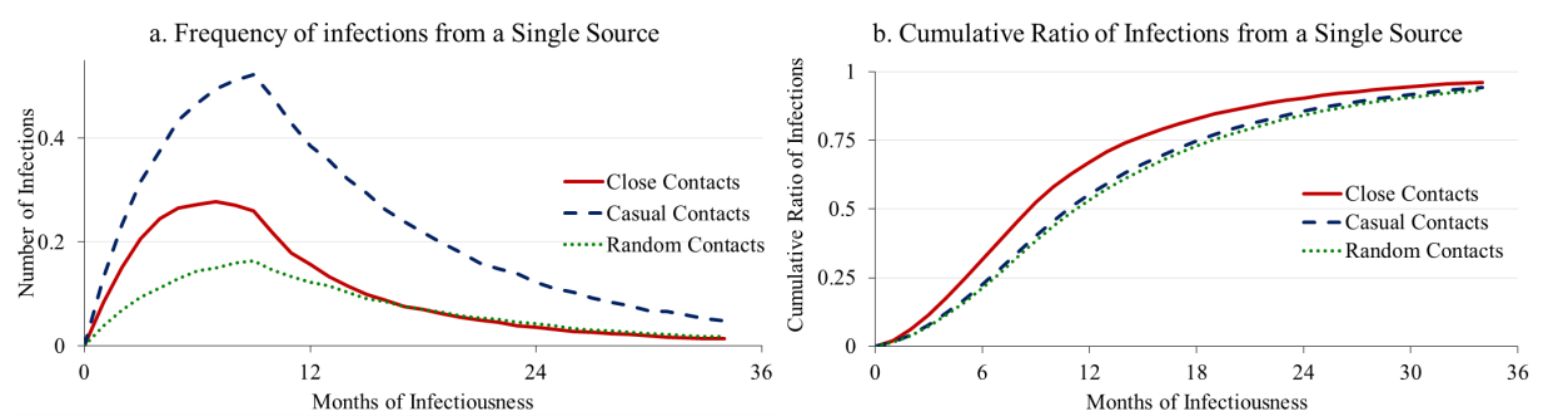

Figure 3: Timing of secondary infections from a single source.

Figure 4 shows the distribution of secondary infections in each network, stratified by type of transmission to an uninfected individual (new infection), versus a previously infected individual (re-infection). New infections show the earliest increase among close contacts due to the high frequency of household contacts. However, as more household members become infected, the number of new infections rapidly declines, and the number of re-infections increases, such that more household infections represent reinfections than new infections after about 12 months of infectiousness, and this ratio continues to grow 
over time (Figure 5). In contrast, casual and random contacts rapidly reach a plateau ratio of new infections to re-infections that remains relatively low, reflecting a large pool of susceptible individuals relative to the number of transmissions occurring.
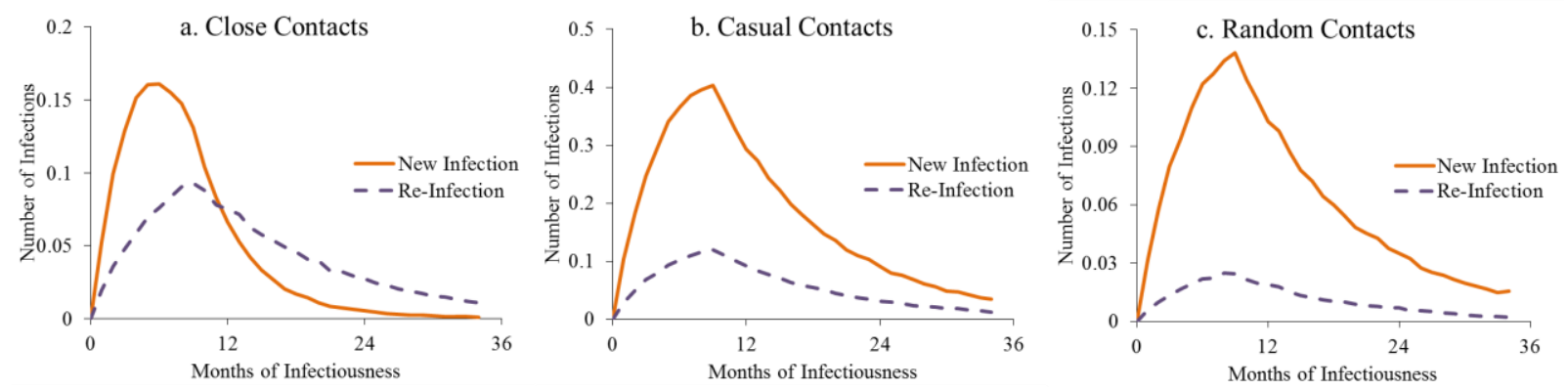

Figure 4: Number of secondary infections stratified by type of infection.

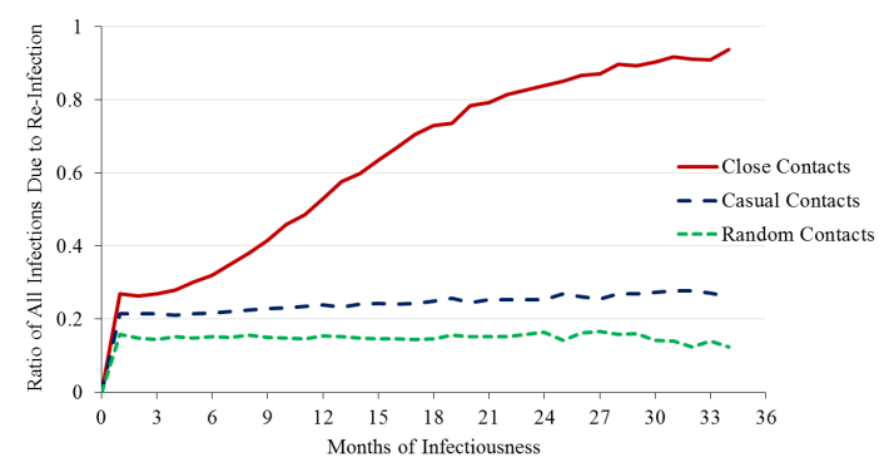

Figure 5: Ratio of all infections representing re-infection.

Finally, we performed a series of one-way sensitivity analyses to ascertain the effect of varying each individual parameter on the time to reach $50 \%$ of transmissions. We varied the duration of casual contacts from 1 to 60 months, the relative sizes of non-household networks by a factor of two in either direction, and the ratio of transmissions in each network (using a three-level factorial experiment with two factors to cover various settings). In each scenario, the effectiveness of contacts were recalibrated to provide the specified ratios of transmission, and the $I_{\max }$ value is tuned to provide the incidence level of 120 per $100000 /$ year. The results show that the time to reach $50 \%$ of transmissions is robust to wide but notunreasonable variation of most parameters, and the only notable effect was observed from increasing the household transmission ratio to a very high level $(80 \%)$, which resulted in a longer duration of 11 months to reach $50 \%$ of transmission in this network.

\section{SUMMARY AND DISCUSSION}

We propose an ABS approach to study the timing and distribution of TB transmissions through the course of the disease. The ABS provides a flexible yet powerful platform to model the population heterogeneity with regard to personal characteristics and different contact networks. Moreover, the ABS ability to represent the course of an epidemic at a micro (individual) level (as well as at a macro population level) enables us to estimate the timing of TB transmissions in our model.

A main implication of the timing and distribution of transmissions is for the development of TB diagnostic interventions. Our results suggest that nearly half of all TB transmissions occur after an individual 
has already been infectious for over one year. Therefore, implementing systems that facilitate diagnosis and treatment of cases in the first year of disease (e.g., annual screening of target populations) can avert a substantial fraction of a population's TB transmission burden. Moreover, our results show that household (close) transmission events occur more quickly in time than transmissions to casual/random contacts. Thus, household-based interventions (e.g., contact tracing) need to occur relatively soon in the infectious course to have maximum impact, whereas population-based interventions (e.g., population screening) may still have important impact if they are widely spaced.

Finally, we detected a remarkably similar pattern for timing of transmissions between non-household contacts, suggesting that there may be a common "shape of transmission over time" outside the household; transmissions to non-household contacts may occur with roughly similar timing regardless of the intensity and duration of contact. This finding may help to simplify future efforts at TB simulation and data collection, by relaxing the requirement to define wide arrays of contact networks, focusing instead on two strata of household vs. non-household (or close vs. other) contacts.

Our work is limited by a relative lack of empirical data on TB transmission and parameter values in the associated contact networks. Our sensitivity analysis of outputs indicates a robust behavior of the results to variation of main parameter values. Nevertheless, additional studies are required to assess the impact of various network structures on timing of transmissions.

Future work include extension of the model to more realistic settings involving multiple interacting communities, age-structured populations, key risk factors (e.g., malnutrition, smoking, poverty), coinfections (especially HIV), and interventions (e.g., household contact tracing) that depend on contact networks and agent's behavior. Subsequent models can also address various policy-making issues such as optimal allocation of TB control resource among multiple populations and interventions (Kasaie and Kelton 2012a, 2012b).

\section{A FAMILY PROBABILITIES OF CHILD BEARING}

A family's probability of child bearing at each month is defined as a decreasing function of the family size, so that the average population size remains constant, and the initial household size distribution keeps a stable shape through the course of the simulation. Using an empirical exponential function (dotted line, Figure 6) to describe the inverse relationship, we tune the individual probabilities for each family size by trial and error. Figure 6 (solid line) shows our final empirical function modeling the decreasing relationship of family size and the probability of child bearing.

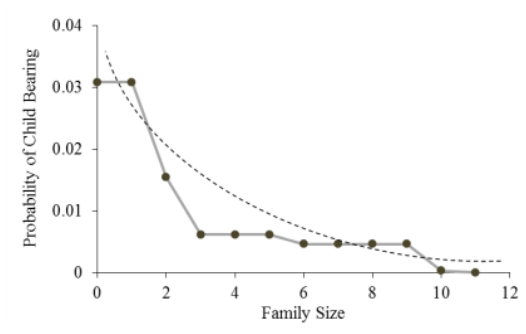

Figure 6: Probability of child bearing for families.

\section{B CONTACT NETWORK CALIBRATION}

Transmission of TB occurs due to contact with an infectious individual, but the probability of transmission depends on several factors, including the duration of exposure, infectiousness of the case, immunity of the contacted individual, etc. Classen et al. (1999) conduct a prospective study in a high-incidence TB setting, and assess the role of social interactions in transmission of TB. Their results show that in such high-incidence areas, contacts outside of the household play an important role in transmission, and ac- 
counts for $70 \%$ of transmissions. Such contacts usually take place during recreation, which in this setting consist of drinking in social groups at a local neighborhood informal bar or at people's homes.

In this sense, we define our contact network in three levels representing the close contacts inside households, and the casual and random contacts outside households. In this definition, random contacts represent short-term encounters with non-related individuals in settings such as transportation, exhibitions, etc. Casual contacts, however, represent a more stable relationship with a smaller population of friends, co-workers, neighbors, etc. who are contacted multiple times for the duration of the relationship. We allow each individual to form his or her networks of contact with a certain likelihood at each time step (e.g., a person may choose to have no random-contact at a given month), and once a network is formed, the members continue to contact each other.

In order to determine the size of each network, we refer to Mossong et al. (2008), who conducts a prospective study on mixing patterns and contact characteristics relevant to the spread of infectious disease. Their results suggest a mean value of 13.4 daily contacts for each person, with $20 \%$ of contacts' occurring at home. We therefore estimate a frequency of 11 daily contacts outside of households, corresponding to 330 contacts in each month. Consequently, we calibrate out contact network parameters as follows:

We let the likelihood of attendance represent the individual's likelihood of forming a contact network. Once a network is formed, it lasts for the duration of contact and the mutual contacts among the network members are executed at each time step. We assume that the probability of attendance in close contacts is 1 (all family members are engaged in a contact), and estimate the likelihood of attendance in casual contacts from Classen et al. (1999). Moreover, we assume that the close contacts have infinite duration (so that the family members are always in contact with each other), while the duration of casual contacts is one year (12 steps) and random contacts last for one month. With regard to the definition of each network, we restrict the domain of close contacts to household members, and casual contacts to neighborhood residents. We let the total casual and random network size be around 330 people (Mossong et al. 2005), and assign about $35 \%$ of these contacts to the casual network (Classen et al. 1999). These assumptions are then used to calibrate the number and size of neighborhoods in our model. Table 2 shows a list of network parameters and their values in the simulation model.

Table 2: List of Contact Network Parameters

\begin{tabular}{llll}
\hline & \multicolumn{2}{c}{ Contact Type } \\
\cline { 2 - 4 } Parameters & Close & Casual & Random \\
\hline Domain (Average Size) & Household (5) & Neighborhood (200) & Community (10200) \\
Duration & Forever & 1 Year & 1 Month \\
Target Network Size & Household Size & 90 & 210 \\
Likelihood of Attendance & 1 & 0.65 & 0.15 \\
Estimated Network size for an Indi- & Household Size & 85 & 230 \\
vidual in Long Term & & & 0.09 \\
Effectiveness of Contacts & 1 & & 0.009 \\
\hline
\end{tabular}

\section{SIMULATION ALIGNMENT AGAINST A DETERMINISTIC MODEL}

We use a simplified instance of the TB ABS model with homogenous mixing, using only random contacts, and align the model against a corresponding deterministic model of TB. The models' parameters are set to the corresponding values in the main simulation model, and the contact rates are calibrated to provide the estimated TB prevalence of 112 per 100,000 person/year. Figure 7 shows the comparison of the TB compartment sizes in both models 
Kasaie, Dowdy, and Kelton
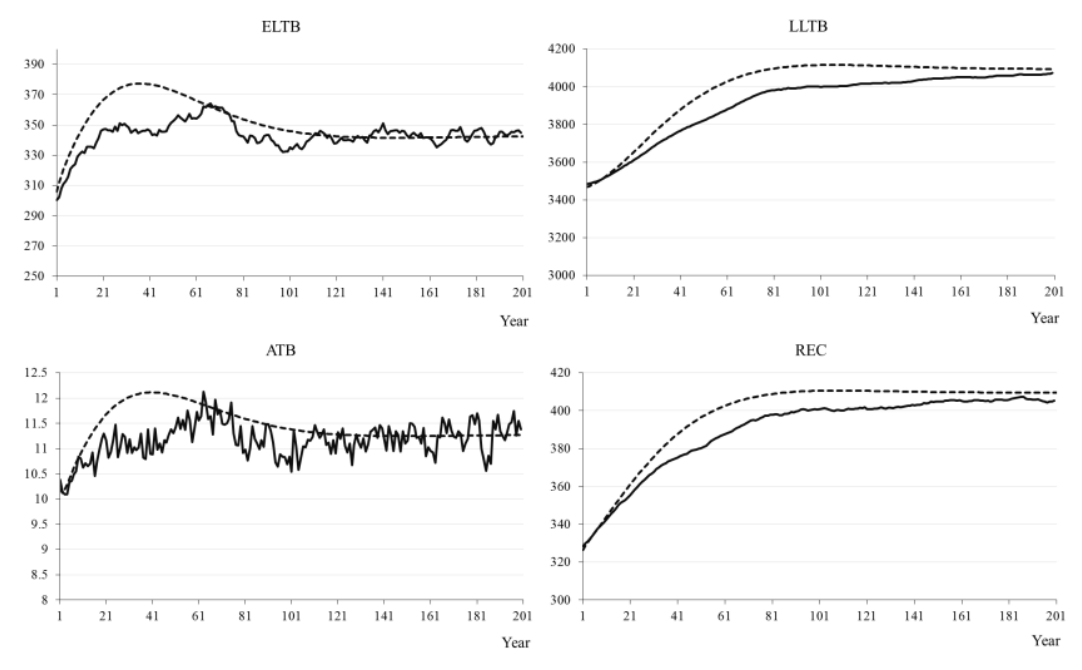

---Deterministic Model — - Homgenous ABS Model

Figure 7: Aligning the homogenous ABS model against the deterministic model.

\section{SIMULATION OUTPUT IN THE BASE SCENARIO}

Table 3 shows the main outputs of the simulation model.

Table 3: ABS Outputs (scale people/year)

\begin{tabular}{lclc}
\hline Output & Mean $+/-\quad 95 \%$ & \multirow{2}{*}{ Output } & $\begin{array}{l}\text { Mean }+/- \\
\text { Half-Width Interval }\end{array}$ \\
\hline Half-Width Interval & $12.24+/-0.33$ & Slow Progression & $1.9+/-0.04$ \\
Prevalence & $11.2+/-0.33$ & ATB Mortality & $1.36+/-0.04$ \\
Duration & $10.94+/-0.05$ & Recovery & $1.34+/-0.04$ \\
Fast Progression & $10.33+/-0.31$ & Treatment & $9.37+/-0.26$ \\
\hline
\end{tabular}

\section{REFERENCES}

Alam, S. J., R. Meyer, and E. Norling. 2008. "A Model for HIV Spread in a South African Village.” In Multi-Agent-Based Simulation IX: International Workshop, by Nuno David and Jaime Simao Sichman. Springer.

Andrews, J. R., F. Noubary, R. P. Walensk, R. Cerda, E. Losina, and C. R. Horsburgh. 2012. "Risk of Progression to Active Tuberculosis Following Reinfection With Mycobacterium Tuberculosis." Clinical Infectious Diseases 54: 784-791.

Atun, R. A., R. M. Lebcir, F. Drobniewski, M. McKee, and R. J. Coker. 2007. "High Coverage With HAART is Required to Substantially Reduce the Number of Deaths from Tuberculosis: System Dynamics Simulation." International Journal of STD and AIDS 18: 267-273.

Brewer, T. F., S. J. Heymann, G. A. Colditz, M. E. Wilson, K. Auerbach, D Kane, and H. V. Fineberg. 1996. "Evaluation of Tuberculosis Control Policies Using Computer Simulation." Journal of the American Medical Association 23: 1898-1903.

Castillo-Chavez, C, and B. Song. 2004. "Dynamical Models of Tuberculosis and Their Applications." Mathematical Bioscience and Engineering 1: 361-404. 
Chao, D. L., M. E. Halloran, V. J. Obenchain, and I. M. Longini. 2010. "FluTE, a Publicly Available Stochastic Influenza Epidemic Simulation Model.” PLoS Computational Biology 6: e1000656.

Classen, C. N., R. Warren, M. Richardson, J. H. Hauman, R. P. Gie, J. HP Ellis, P. D. van Helden, and N. Beyers. 1999. "Impact of Social Interactions in the Community on the Transmission of Tuberculosis in a High Incidence Area." Thorax 54: 136-140.

CORE Group. 2006. Tuberculosis Control Programming for PVOs: Facilitator's Manual. Washington, D.C. Accessed March 13. http://pdf.usaid.gov/pdf_docs/PNADG190.pdf.

Dowdy, D.W., C. Dye, and T. Cohen. 2012. "Data Needs for Evidence-Based Decisions: A TB Modeler's Wish List." International Journal of Tuberculosis and Lung Disease. In Press.

Dowdy, D. W., J. E. Golub, R. A. Chaisson, and V. Saracenic. 2012. "Heterogeneity in Tuberculosis Transmission and the Role of Geographic Hotspots in Propagating Epidemics." Proceedings of the National Academy of Sciences 109: 9557-9562.

Epstein, J. M. 1999. "Agent-Based Computational Models And Generative Social Science.” Complexity 4: 41-60.

Espíndola, A. L., C.T. Bauch, B. C. T. Cabella, and A. S. Martinez. 2011. "An Agent-Based Computational Model of the Spread of Tuberculosis." Journal of Statistical Mechanics: Theory and Experiment 5: P05003.

Google. Public Data: Life Expectancy. 2010. Accessed March 13, 2012. https://www.google.com/publicdata/explore?ds=d5bncppjof8f9_\&met_y=sp_dyn_le00_in\&idim=cou ntry:USA\&dl=en\&hl=en\&q=average $\% 201$ ife $\% 20$ expectancy $\#$ ! ctype $=1 \&$ strail $=$ false $\& b c s=d \& n s e l m=$ $h \& m e t \_y=s p \_d y n \_l e 00 \_$in\&scale_y=lin\&ind_y=false\&rdim=region\&idim=region:MNA:SSA:SA .

Horsburgh, C. R. 2004. "Priorities for the Treatment of Latent Tuberculosis Infection in the United States." New England Journal of Medicine 350: 2060-2067.

Hughes, G. R., C. S. M. Currie, and E. L. Corbett. 2006. "Modeling Tuberculosis In Areas of High HIV Prevalence." In Proceedings of the 2006 Winter Simulation Conference, edited by F. P. Wieland, J. Liu, B. G. Lawson, D. M. Nicol, and R. M. Fujimoto, eds L. F. Perrone, 459-465. Piscataway, New Jersey: Institute of Electrical and Electronics Engineers, Inc.

Kasaie, P., and W. D. Kelton. 2012a. "Resource Allocation for Controlling Epidemics: Calibrating, Analyzing, and Optimizing an Agent-Based Simulation." IIE Transactions on Healthcare Systems Engineering. In Press.

Kasaie, P., and W. D. Kelton. 2012b. "Simulation Optimization for Allocation of Epidemic-Control Resources." IIE Transactions on Healthcare Systems Engineering. In Press.

Langley, I., B. Doulla, H. Lin, K. Millington, and B. Squire. 2012. "Modeling the Impacts of New Diagnostic Tools for Tuberculosis in Developing Countries to Enhance Policy Decisions." Health Care Management Science 21: 1-15.

Longini, I. M., M. E. Halloran, A. Nizam, Y. Yang, S. Xu, D. S. Burke, D. AT Cummings, and J. M. Epstein. 2006. "Containing a Large Bioterrorist Smallpox Attack: A Computer Simulation Approach." International Journal of Infectious Diseases 11: 98-108.

Macal, C. M., and M. J. North. 2010. "Tutorial on Agent-Based Modeling and Simulation." Journal of Simulation 4: 151-162.

Mellor, G. R., C. S. M. Currie, and E. L. Corbett. 2011. "Incorporating Household Structure into a Discrete-Event Simulation Model of Tuberculosis and HIV." ACM Transactions on Modeling and Computer Simulation 21: 26.

Mossong, J., N. Hens, M. Jit, P. Beutels, K. Auranen, R. Mikolajczyk, M. Massari, S Salmaso, G. S. Tomba, J. Wallinga, J. Heijne, M. Sadkowaska-Todys, M. Rosinska, W. J. Edmunds. 2008. "Social Contacts and Mixing Patterns Relevant to the Spread of Infectious Diseases." PLoS medicine 5: e74.

Porco, T. C., and S. M. Blower. 1998. "Quantifying the Intrinsic Transmission Dynamics of Tuberculosis." Theoretical Population Biology 54: 117-132. 
Raffalli, J., K. A. Sepkowitz, and D. Armstrong. 1996. "Community-Based Outbreaks of Tuberculosis." JAMA International Medicine 156: 1053-1060.

Tiemersma, Edine W., M. J. van der Werf, M. W. Borgdorff, B. G. Williams, and N. GD. Nagelkerke. 2011. "Natural History of Tuberculosis: Duration and Fatality of Untreated Pulmonary Tuberculosis in HIV Negative Patients: A Systematic Review." PLoS One 6: e17601.

United Nations Data Retrieval System. 2013. Households by age and sex of reference person and by size of household. UNSD Demographic Statistics | United Nations Statistics Division. Accessed March 13. http://data.un.org/Data.aspx?d=POP\&f=tableCode:50 .

Verver, S., R. M. Warren, Z. Munch, M. Richardson, G. D. van der Spuy, M. W. Borgdorff, M. A. Behr, N. Beyers, and P. D. van Helden. 2004. "Proportion of Tuberculosis Transmission That Takes Place in Households in a High-Incidence Area." Lancet 363: 212-214.

Vynnycky, E., and P. Fine. 1997. "The Natural History of Tuberculosis: The Implications of Age Dependent Risks of Disease and the Role of Reinfection." Epidemiology and Infection 119: 183-201.

Waaler, H. T., A. Gese, and S. Anderson. 1962. "The Use of Mathematical Models in the Study of the Epidemiology of Tuberculosis." American Journal of Pubic Health 52: 1002-1003.

WHO. 2012.Global Tuberculosis Report 2012. World Health Organization.

XJ Technologies. 2012. "AnyLogic University 6.8.1."

\section{AUTHOR BIOGRAPHIES}

PARASTU KASAIE is a PhD candidate in Operations, Business Analytics and Information Systems, and a M.S. student in Biostatistics, at the University of Cincinnati. Her research interest is in simulation modeling and statistical analysis of infectious diseases. Her email address is kasaie.parastu@gmail.com.

DAVID W. DOWDY is the B. Frank and Kathleen Polk Assistant Professor of Epidemiology at the Johns Hopkins Bloomberg School of Public Health. His research interests include epidemiological investigation, economic evaluation, and mathematical modeling of tuberculosis and TB/HIV. He is particularly interested in the evaluation of diagnostic tests for TB and other infectious diseases and in development of "user-friendly" models for translation of findings to individuals without modeling expertise. Dr. Dowdy serves as a Steering Committee member of the TB Modeling and Analysis Consortium (funded by the Bill and Melinda Gates Foundation), modeling core director of the TB Clinical Diagnostics Research Consortium (funded by the U.S. National Institutes of Health), and chair of the Modeling Subgroup of the TB Diagnostics Research Forum (co-funded by both institutions). His email address is ddowdy@jhsph.edu.

W. DAVID KELTON is a Professor in the Department of Operations, Business Analytics and Information Systems at the University of Cincinnati, and Director of the MS - Business Analytics Program there. His research interests and publications are in the probabilistic and statistical aspects of simulation, applications of simulation, and stochastic models. He is co-author of Simio and Simulation: Modeling, Analysis, and Applications, as well as Simulation with Arena, and was also coauthor of the first three editions of Simulation Modeling and Analysis, all for McGraw-Hill. He was Editor-in-Chief of the INFORMS Journal on Computing from 2000-2007. He served as WSC Program Chair in 1987, General Chair in 1991, was on the WSC Board of Directors from 1991-1999, and is a Founding Trustee of the WSC Foundation. He is a Fellow of both INFORMS and IIE. His email address is david.kelton@uc.edu. 